\title{
Quantitative Measurement Methods for Acute Respiratory Distress Syndrome
}

\author{
Shimeng $\mathrm{Ji}^{1}$ and Yuanlin Song ${ }^{1,2,3}$ \\ ${ }^{1}$ Department of Pulmonary Medicine, Fudan University, China. \\ ${ }^{2}$ Shanghai Public Health Clinical Center, Fudan University, China \\ ${ }^{3}$ Zhongshan Hospital, Qingpu branch, Fudan University, China
}

Submission: February 18, 2017; Published: May 31, 2017

*Corresponding author: Yuanlin Song, Department of Pulmonary Medicine, Zhongshan Hospital, Fudan University, China, Tel: +86 21 64041990x2422; Fax: +86 21 54961729; Email: ylsong70@gmail.com

\begin{abstract}
Acute respiratory distress syndrome (ARDS) is characterized by the presence of hypoxia not fully explained by cardiac failure or fluid overload and bilateral pulmonary infiltrates on chest imaging. The syndrome has mortality varying from $20 \%$ to $40 \%$ and emphasizes medical burden globally. Therefore the early recognition and quantification of lung edema is important to establish the correct treatment. Several investigational modalities are potentially helpful in recognition of ARDS, such as chest radiography, chest computed tomography, thoracic ultrasonography, thermo dilution etc., among them noninvasive imaging measurements have been widely used because of their accessibility and convenience. This review aimed to examine the effectiveness of three noninvasive imaging measurement methods in diagnosis of ARDS, especially their predictive effect on severity of ALI/ARDS.
\end{abstract}

Keywords: Quantitative Measurement; Acute Respiratory Distress Syndrome; Lung Edema

\section{Introduction}

Acute respiratory distress syndrome (ARDS) is a form of acute progressive respiratory failure with non-cardiogenic pulmonary edema secondary to inflammation process, which can be either pulmonary or non-pulmonary origin. Interstitial lung edema, diffused alveolar damage and alveolar hyaline membrane formed are the most pathologic characteristics of ARDS [1]. Clinical manifestation includes dyspnea and refractory hypoxemia. Since the first description published by Ashbaugh and colleagues in year of 1967 [2], the definition has been revised three times with the improvement of recognition of the syndrome. In 2012, a new definition, namely Berlin definition was published and widely used thereafter [3]. The improved ARDS definition is described as new respiratory failure, or worsening of chronic respiratory disease within 7 days not fully explained by cardiac failure or fluid overload; chest imaging (Chest X-ray or CT scan) for the demonstration of lung opacities, not fully explained by effusions, lobar/lung collapse, or nodules; a minimum positive end-expiratory pressure (PEEP) of $5 \mathrm{~cm} \mathrm{H} 20$. Meanwhile, the new definition classified the syndrome as mild, moderate and severe based on oxygenation criteria. Although the Berlin definition is an evolution of the previous definitions, it still has its limitations. Overall, one-third of patients with ARDS was under-recognized by clinicians and is significantly delayed in their treatment [4]. Thus, timely recognition and stratification of ARDS appears critical, as it is associated with early mechanic ventilation, low tidal volume ventilation, prone positioning which can lower mortality [5].

Lung edema is the most important pathological character of ARDS [6]. Quantitative measurement of lung edema can act as an ideal predictor of disease severity and prognosis $[7,8]$. Thermo dilution has become a standard instrument to measure extra vascular lung water index (EVLWI) in many intensive care units (ICUs) and it has been shown that even small changes in EVLW can be detected [7,9-12]. However, the requirement of placing intravascular catheters makes it costly, risky and invasive. For these reasons, we reviewed previous studies to examine the utility and effectiveness of accessible and non-invasive imaging methods to quantitative measure lung water and predict the prognosis of ARDS.

\section{Chest Radiography}

In the original definition of ARDS, bilateral pulmonary infiltrates in the absence of left atrial hypertension of anteriorposterior chest X-ray is a necessary criterion for the diagnosis 
of ARDS [6]. Chest radiography is a bedside available and timesaving measurement that has been used widely to access EVLW in ICU, but usually its sensitivity and specificity are largely uncertain as it cannot discriminate pulmonary infiltrates between cardiogenic and non-cardiogenic lung edema and in some circumstances ARDS can be diagnosed in the presence of cardiac failure $[13,14]$. In addition, the inter-observer variability is also significant: even between two radiographic experts, interpretations may be different depending on their own clinical experience and a long period of training program is needed for minimization of the variability from inter-observers $[15,16]$.

Figueroa-Casas et al. evaluated the accuracy of the chest radiograph using computed tomography as reference standard in ninety patients with ARDS [17]. The radiograph images were analyzed by two independent radiologists. They found the raw agreement for the presence of bilateral findings for chest radiograph consistent with ARDS was much lower than that for CT scanning and the sensitivity and specificity for chest X-ray was only 0.73 and 0.70 , respectively. These findings suggest that the inter-observer agreement for the chest X-ray is quite low which mainly results in under- or over-diagnosis of the syndrome. To improve the effectiveness of diagnosis of ARDS by chest radiography, another study group introduced a computer aided chest radiographs diagnosis system for ARDS based on texture analysis of inter costal patches selected automatically [18]. The computer-aided diagnosing method evaluated on 90 radiographs among which 53 images present ARDS and the sensitivity was $90.6 \%$ at a specificity of $86.5 \%$. Even though with the help of computer the sensitivity of X-ray, which is more preferred by intensivists, has been improved, the disadvantages of chest radiography cannot be avoided such as exposure to radiation and long waits for radiography to be conducted which leads to significant delay in decision-making. For these reasons, increasing questions have been raised about the utility of the chest radiography in the diagnosis of ARDS [4].

\section{Thoracic Ultrasonography}

Thoracic ultrasonography has been increasingly used as an excellent tool to assess EVLW and may help to discriminate rapidly between cardiogenic and non-cardiogenic causes of dyspnea [13]. In an ultrasound image, A lines are repetitive horizontal artifacts at regular intervals parallel to the pleural line. B lines are discrete vertical hyperchoic comet-tail artifacts arising from the pleural surface to the bottom of the screen without fading [19]. In previous studies, B lines were found to indicate interstitial edema and confluent B lines to suggest alveolar edema [20].

Thiago M. Santos et al. prospectively evaluated 6 chest regions by ultrasound on 61 sepsis patients in their emergency department using a simplified lung edema scoring system (SLESS) to assess the effect of the lung edema in the gas exchange [19]. After comparison of SLESS with the Mortality in Emergency
Department Sepsis Score (MEDS score) and the third version of the Simplified Acute Physiology Score scoring systems (SAPS3 score), they concluded that SLESS had a good correlation with MEDS and SAPS3 score and a negative correlation with the $\mathrm{PaO}_{2} /$ $\mathrm{FiO}_{2}$ ratio. What's more, for the patients who had pulmonary original infections, they had significantly higher SLESS scores than those who had non pulmonary infections. Philipp Enghard and colleagues also reported promising results about the EVLW assessment by ultra sound but using a simplified four-region protocol [21]. The ultrasound scoring system was based on the number of single and confluent B lines per inter costal. Fifty ventilated patients in ICU underwent the simplified thoracic ultrasound as well as transpulmonary thermo dilution measurement, chest radiography and central venous pressure (CVP) measurements to compare the utility of different methods for EVLWI. They found the simplified ultrasound protocol and the scoring system had an excellent correlation with EVLWI measured by thermo dilution but the correlation between ultrasound score and CVP was not significant; in addition, the correlation between chest radiography and EVLWI was significant but rather weak. Ultrasound is a noninvasive, convenient and fast approach to diagnose and evaluate the severity of lung edema in critical patients, but the use of it as part of routine examination in ARDS patients is not widespread and no consensus has been reached yet.

\section{Computed Tomography}

Since the CT features of ARDS were first described in the 1980 s [22,23], the quantitative analysis of CT scanning was used as a pivotal tool in the understanding of the pathophysiology of ARDS and provided a firm rationale for mechanical ventilation setting $[24,25]$. According to radiographic attenuation values, the entire lung was divided into four compartments: hyper inflated, radiographic attenuation values between -1000 and $-900 \mathrm{HU}$; normal aerated, radiographic attenuation -500 to $-900 \mathrm{HU}$; poorly aerated, radiographic attenuation -100 to $-500 \mathrm{HU}$, and no aerated or "gasless", radiographic attenuation - 100 to $100 \mathrm{HU}$ [26-29]. Assuming the attenuation $+1000 \mathrm{HU}$ approximates the physical density of bone, $-1000 \mathrm{HU}$ corresponds to the density of air, and $0 \mathrm{HU}$ approximates water's physical density, it is possible to compute the volume of the entire lung or the particular interest region of the lung and the volume of gas. Therefore, recognition the loss of lung aeration in ARDS patients can help to speed evaluate the amount of lung water outside the pulmonary vasculature [30-32]. Previous studies have demonstrated that the accuracy of the quantitative CT analysis in measuring extra vascular lung water showed good agreement with the gravimetric methods, a gold standard method at autopsy, and also had a good correlation with single-indicator thermo dilution $[28,33]$.

One to three CT scan slides instead of scanning the overall lung taken at different airway pressure levels are sufficient to quantified precisely lung recruit ability, which is critical to guide mechanical ventilation setting [5,34]. In clinical trials, tidal 
volume was standardized with body weight to assess the lung volume but there was no evidence of relationship between body weight and the portion of the lung open to ventilation $[5,35,36]$. Patients who share the same body weight could have different lung recruit ability. If a high tidal volume is applied on the patient whose portion of lung open to ventilation is limited, the alveolar strain would be high enough to cause further lung injury. On the contrary, for the patient with high lung recruitment, a too low tidal volume would fail to open the portion of the lung parenchyma that could ventilate. So the tidal volume should be set in accordance with actual portion of lung parenchyma open to ventilation to prevent further lung injury caused by mechanical ventilation.

It is acknowledged that applying a higher PEEP is beneficial to prevent local excessive stress and strain on the structures at the interface between the closed and the open portion of the lung [37]. Indeed, depending on the lung recruit ability measured by quantitative CT analysis, a proper PEEP value can be set to maintain open lung regions that have been opened during inspiration but that would otherwise collapse at end-expiration $[30,38,39]$. Meanwhile, CT scanning helps to assess and monitor the response to mechanical ventilation but also timely reveal complications, such as pleural effusion, lobar atelectasis and pneumothorax [26,40,41]. Although CT scanning has some disadvantages such as unavoidable ionizing radiation exposure and transport risk to mobilize critically ill patients to CT facility, quantitative CT analysis has become a standard method to quantify different aerated conditions of the lung parenchyma and to evaluate lung recruit ability.

\section{Summary}

Despite promising preclinical and early clinical data, acute respiratory distress syndrome still has a high mortality. The most important issue is under recognition of ARDS. Although the current imaging techniques have provided quite much information about local pathological changes, anatomic data and response to treatment in patients with ARDS, the speed and precise quantification of lung edema and the severity of the syndrome is limited. Computed tomography may be a desirable tool but the ionizing radiation and transportation risk make it cannot be used frequently as a routine examination. Therefore, the current quantitative measurement methods for ARDS need to evolve and new methods should be proposed to improve ARDS recognition and evaluation.

\section{References}

1. Weinacker AB, Vaszar LT (2001) acute respiratory distress syndrome: physiology and new management strategies. Annual review of medicine 52: 221-237.

2. Ashbaugh DG, Bigelow DB, Petty TL, Levine BE (1967) acute respiratory distress in adults. Lancet 2(7511): 319-323.

3. Force ADT, Ranieri VM, Rubenfeld GD, Thompson BT, Ferguson ND, et al. (2012) acute respiratory distress syndrome: the Berlin Definition. Jama 307(23): 2526-2533.
4. Laffey JG, Pham T, Bellani G (2017) Continued under-recognition of acute respiratory distress syndrome after the Berlin definition: what is the solution? Current opinion in critical care 23(1): 10-17.

5. Gattinoni L, Caironi P, Valenza F, Carlesso E (2006) the role of CT-scan studies for the diagnosis and therapy of acute respiratory distress syndrome. Clin Chest Med 27(4): 559-570.

6. Bernard GR, Artigas A, Brigham KL, Carlet J, Falke K, et al. (1994) The American-European Consensus Conference on ARDS. Definitions, mechanisms, relevant outcomes, and clinical trial coordination. American journal of respiratory and critical care medicine 149(3 Pt 1): $818-824$.

7. Sakka SG, Klein M, Reinhart K, Meier-Hellmann A (2002) Prognostic value of extra vascular lung water in critically ill patients. Chest 122(6): 2080-2086.

8. Kuzkov VV, Kirov MY, Sovershaev MA, Kuklin VN, Suborov EV, et al. (2006) Extra vascular lung water determined with single transpulmonary thermo dilution correlates with the severity of sepsisinduced acute lung injury. Critical care medicine 34(6): 1647-1653.

9. Dres M, Teboul JL, Guerin L, Anguel N, Amilien V, et al. (2014) Transpulmonary thermo dilution enables to detect small short-term changes in extra vascular lung water induced by a bronchoalveolar lavage. Crit Care Med 42(8): 1869-1873.

10. Brown LM, Liu KD, Matthay MA (2009) Measurement of extra vascular lung water using the single indicator method in patients: research and potential clinical value. Am J Physiol Lung Cell Mol Physiol 297(4): L547-558.

11. Rossi P, Wanecek M, Rudehill A, Konrad D, Weitzberg E, et al. (2006) Comparison of a single indicator and gravimetric technique for estimation of extra vascular lung water in endo toxemic pigs. Crit Care Med 34(5):1437-1443.

12. Jozwiak M, Silva S, Persichini R, Anguel N, Osman D, et al. (2013) Extra vascular lung water is an independent prognostic factor in patients with acute respiratory distress syndrome. Crit Care Med 41(2): 472480 .

13. Cibinel GA, Casoli G, Elia F, Padoan M, Pivetta E, et al. (2012) Diagnostic accuracy and reproducibility of pleural and lung ultrasound in discriminating cardiogenic causes of acute dyspnea in the emergency department. Intern Emerg Med 7(1): 65-70.

14. Blanco PA, Cianciulli TF (2016) Pulmonary Edema Assessed by Ultrasound: Impact in Cardiology and Intensive Care Practice. Echocardiography 33(5): 778-787.

15. Rubenfeld GD, Caldwell E, Granton J, Hudson LD, Matthay MA, et al. (1999) Interobserver variability in applying a radiographic definition for ARDS. Chest 116(5): 1347-1353.

16. Meade MO, Cook RJ, Guyatt GH, Groll R, Kachura JR, et al. (2000) Interobserver variation in interpreting chest radiographs for the diagnosis of acute respiratory distress syndrome. Am J Respir Crit Care Med 161(1): 85-90.

17. Figueroa-Casas JB, Brunner N, Dwivedi AK, Ayyappan AP (2013) Accuracy of the chest radiograph to identify bilateral pulmonary infiltrates consistent with the diagnosis of acute respiratory distress syndrome using computed tomography as reference standard. J Crit Care 28(4): 352-357.

18. Zaglam N, Jouvet P, Flechelles O, Emeriaud G, Cheriet F, et al. (2014) Computer-aided diagnosis system for the Acute Respiratory Distress Syndrome from chest radiographs. Comput Biol Med 52: 41-48.

19. Santos TM, Franci D, Coutinho CM, Ribeiro DL, Schweller M, et al. (2013) A simplified ultrasound-based edema score to assess lung injury and clinical severity in septic patients. Am J Emerg Med 31(12): 1656-1660. 
20. Bouhemad B, Liu ZH, Arbelot C, Zhang M, Ferarri F, et al. (2010) Ultrasound assessment of antibiotic-induced pulmonary reaeration in ventilator-associated pneumonia. Critical care medicine 38(1): 84-92.

21. Enghard P, Rademacher S, Nee J, Hasper D, Engert U, et al. (2015) Simplified lung ultrasound protocol shows excellent prediction of extravascular lung water in ventilated intensive care patients. Crit Care 19: 36.

22. Gattinoni L, Mascheroni D, Torresin A, Marcolin R, Fumagalli R, et al (1986) Morphological response to positive end expiratory pressure in acute respiratory failure. Computerized tomography study. Intensive Care Med 12(3): 137-142.

23. Maunder RJ, Shuman WP, McHugh JW, Marglin SI, Butler J, et al. (1986) Preservation of normal lung regions in the adult respiratory distress syndrome. Analysis by computed tomography. Jama 255(18): 24632465.

24. Gattinoni L, Presenti A, Torresin A, Baglioni S, Rivolta M, et al. (1986) Adult respiratory distress syndrome profiles by computed tomography. J Thorac Imaging 1(3): 25-30.

25. Gattinoni L, Pelosi P, Pesenti A, Brazzi L, Vitale G, et al. (1991) CT scan in ARDS: clinical and physiopathological insights. Acta anaesthesiologica Scandinavica Supplementum 95: 87-94.

26. Rouby JJ, Puybasset L, Nieszkowska A, Lu Q (2003) acute respiratory distress syndrome: lessons from computed tomography of the whole lung. Crit Care Med 31(4 Suppl): S285-295.

27. Malbouisson LM, Muller JC, Constantin JM, Lu Q Puybasset L, et al. (2001) Computed tomography assessment of positive end-expiratory pressure-induced alveolar recruitment in patients with acute respiratory distress syndrome. Am J Respir Crit Care Med 163(6): 1444-1450.

28. Zhang F, Li C, Zhang JN, Guo HP, Wu DW, et al. (2014) Comparison of quantitative computed tomography analysis and single-indicator thermo dilution to measure pulmonary edema in patients with acute respiratory distress syndrome. Biomed Eng Online 13:30.

29. Puybasset L, Cluzel P, Chao N, Slutsky AS, Coriat P, et al. (1998) A computed tomography scan assessment of regional lung volume in acute lung injury. The CT Scan ARDS Study Group. Am J Respir Crit Care Med 158(5 Pt 1):1644-1655.

30. Caironi P, Carlesso E, Gattinoni L (2006) Radiological imaging in acute lung injury and acute respiratory distress syndrome. Semin Respir Crit Care Med 27(4): 404-415.
31. Waerhaug K, Kuzkov VV, Kuklin VN, Mortensen R, Nordhus KC, et al. (2009) Inhaled aerosolized recombinant human activated protein $C$ ameliorates endotoxin-induced lung injury in anaesthetised sheep. Critical care 13(2): R51.

32. Patroniti N, Bellani G, Maggioni E, Manfio A, Marcora B, et al. (2005) Measurement of pulmonary edema in patients with acute respiratory distress syndrome. Crit Care Med 33(11): 2547-2554.

33. Kuzkov VV, Suborov EV, Kirov MY, Waerhaug K, Mortensen R, et al. (2010) Radiographic lung density assessed by computed tomography is associated with extra vascular lung water content. Acta Anaesthesiol Scand 54(8): 1018-1026.

34. Gattinoni L, Caironi P, Pelosi P, Goodman LR (2001) What has computed tomography taught us about the acute respiratory distress syndrome? Am J Respir Crit Care Med 164(9): 1701-1711.

35. Brower RG, Matthay MA, Morris A, Schoenfeld D, Thompson BT, et al. (2000) Ventilation with lower tidal volumes as compared with traditional tidal volumes for acute lung injury and the acute respiratory distress syndrome. The Acute Respiratory Distress Syndrome Network. N Engl J Med 342(18): 1301-1308.

36. Brochard L, Roudot-Thoraval F, Roupie E, Delclaux C, Chastre J, et al. (1998) Tidal volume reduction for prevention of ventilator-induced lung injury in acute respiratory distress syndrome. The Multicenter Trail Group on Tidal Volume reduction in ARDS. Am J Respir Crit Care Med 158(6): 1831-1838.

37. Muscedere JG, Mullen JB, Gan K, Slutsky AS (1994) Tidal ventilation at low airway pressures can augment lung injury. Am J Respir Crit Care Med 149(5): 1327-1334.

38. Crotti S, Mascheroni D, Caironi P, Pelosi P, Ronzoni G, et al. (2001) Recruitment and de recruitment during acute respiratory failure: a clinical study. Am J Respir Crit Care Med 164(1): 131-140.

39. Pelosi P, Goldner M, McKibben A, Adams A, Eccher G, et al. (2001) Recruitment and de recruitment during acute respiratory failure: an experimental study. Am J Respir Crit Care Med 164(1): 122-130.

40. Terragni PP, Rosboch G, Tealdi A, Corno E, Menaldo E, et al. (2007) Tidal hyperinflation during low tidal volume ventilation in acute respiratory distress syndrome. Am J Respir Crit Care Med 175(2): 160-166.

41. Caironi P, Cressoni M, Chiumello D, Ranieri M, Quintel M, et al. (2010) Lung opening and closing during ventilation of acute respiratory distress syndrome. Am J Respir Crit Care Med 181(6): 578-586.

\begin{tabular}{l} 
Your next submission with Juniper Publishers \\
will reach you the below assets \\
- Quality Editorial service \\
- Swift Peer Review \\
- Reprints availability \\
- E-prints Service \\
- Manuscript Podcast for convenient understanding \\
- Global attainment for your research \\
- Manuscript accessibility in different formats \\
( Pdf, E-pub, Full Text, Audio) \\
- Unceasing customer service \\
Track the below URL for one-step submission \\
https://juniperpublishers.com/online-submission.php \\
\hline
\end{tabular}

